\title{
Aulas de Educação Física e indicadores de violência em adolescentes
}

\section{Physical Education classes and violence indicators among adolescents}

\author{
Simone Storino Honda Barros ${ }^{1,2}$ \\ Mauro Virgílio Gomes de Barros ${ }^{1,2}$ \\ Carla Menêses Hardman ${ }^{1,2}$ \\ Agostinho Gonçalves da Silva Júnior ${ }^{2}$ \\ Juarez Vieira do Nascimento ${ }^{1}$ \\ Anísio Luiz da Silva Brito ${ }^{2}$ \\ Markus Vinicius Nahas ${ }^{1}$
}

\section{Resumo}

O objetivo deste estudo transversal foi analisar a associação entre a participação nas aulas de Educação Física (EF) e indicadores de violência em estudantes. Um questionário foi usado para coletar dados pessoais, da participação nas aulas de EF e de indicadores de violência em 4.210 adolescentes (14-19 anos) estudantes do ensino médio do estado de Pernambuco, Brasil. A prevalência de "envolvimento em brigas" e de "envolvimento em episódios de violência física como vitima” foi, respectivamente, de $21,2 \%$ e $10,7 \%$. Verificou-se que $64,9 \%$ dos estudantes relataram não participar das aulas de EF. A prevalência dos indicadores de violência foi maior entre os rapazes, enquanto a não participação nas aulas de EF foi maior entre as moças. Independentemente do sexo e de outras covariáveis, verificou-se que a participação nas aulas de EF foi um fator diretamente associado a envolvimento em brigas. Concluiu-se que a participação nas aulas de EF está associada a envolvimento em brigas em estudantes adolescentes, contrariando, neste caso específico, a hipótese de que a participação nessas aulas está associada a condutas de saúde.

\section{Palavras-chave}

Educação física; Violência; Adolescentes; Conduta de saúde; Estudo transversal.

\begin{abstract}
The aim of this cross-sectional study was to analyze the association between enrollment in physical education classes and violence indicators among students. A questionnaire was used to gather personal data, participation in physical education lessons, and violence indicators from 4,210 adolescents (1419 years) bigh school students from the state of Pernambuco, Brazil. The prevalence of involvement in physical fights and being victim of physical violence was, respectively, of $21.2 \%$ and $10.7 \%$. It was verified that $64.9 \%$ of the students reported have not been enrolled in physical education classes. The prevalence of violence indicators was higher among males while no enrollment in PE classes was higher among females. Independently of gender and other covariates, it was verified that enrollment in $P E$ classes was directly associated with physical fights. It was concluded that enrollment in PE classes is associated with physical fights among adolescents students, which contradicts, in this particular, the hypothesis that participation in PE classes is associated with positive health behaviors.
\end{abstract}

\section{Keywords}

Physical education. Violence. Adolescents. Health behavior. Cross-sectional study.
Rev Bras Ativ Fis Saúde p. 566-575 DOI:

http://dx.doi.org/10.12820/rbafs.v.18n5p566

1 Programa de Pós-graduação em Educação Física, Universidade Federal de Santa Catarina, Florianópolis, SC, Brasil.

2 Programa Associado de Pós-graduação em Educação Física UPE/UFPB, Grupo de Pesquisa em Estilos de Vida e Saúde (GPES), Escola Superior de Educação Física, Universidade de Pernambuco, Recife, Brasil. 


\section{INTRODUÇÃO}

A participação em atividades físicas e esportes vem sendo apontada como um fator de proteção em relação ao estresse, aos sintomas de depressão e o uso de drogas em adolescentes ${ }^{1}$. Por outro lado, há também evidências ligando a prática de atividades físicas a uma maior chance de consumo de bebidas alcoólicas neste subgrupo populacional ${ }^{2}$.

Tendo em vista que atividades físicas e práticas esportivas representam conteúdos importantes das aulas de Educação Física, a hipótese de que a participação nestas aulas está associada a condutas de saúde em adolescentes vem sendo examinada em diversos estudos ${ }^{3-5}$. De modo geral, estas investigações evidenciaram que participar das aulas de Educação Física é um fator associado ao nível de atividade física global e aos hábitos alimentares de estudantes adolescentes.

Particularmente entre os jovens e nos países em desenvolvimento como o Brasil, a violência representa uma das principais causas de incapacidade e morte . $^{6}$ Apesar de representar um importante problema de saúde pública, a violência física interpessoal é um tema que tem recebido limitada atenção no âmbito da intervenção e da pesquisa ${ }^{7}$, havendo escassez de dados sobre os fatores sociais, culturais e demográficos que estão associados ou determinam a ocorrência dos episódios de violência física em adolescentes.

O estudo recente conduzido por Taliaferro, Rienzo e Donovan $(2010)^{1}$ evidenciou que a prática de esportes organizados é um fator determinante de muitas condutas relacionadas à saúde, mas está positivamente correlacionada ao envolvimento em brigas na escola. Outros estudos reforçam este achado, evidenciando que estudantes adolescentes que praticam esportes em comparação aos não praticantes apresentaram maior chance de carregar armas para escola e de se envolver em brigas $^{8-10}$.

O presente estudo foi delineado a partir de três considerações fundamentais: primeiro, no sistema educacional brasileiro os esportes são um conteúdo tradicional das aulas de Educação Física ${ }^{11}$; segundo, as aulas de Educação Física podem assumir um papel de destaque no desenvolvimento de intervenções em saúde ${ }^{12}$; e, terceiro, há escassez de evidências sobre os fatores que estão associados à violência física na adolescência ${ }^{7}$, limitando o desenvolvimento da pesquisa e de intervenções efetivas para lidar com este importante problema de saúde pública. Assim, o objetivo do presente estudo foi analisar a associação entre a participação nas aulas de Educação Física e indicadores de violência em adolescentes estudantes do ensino médio.

\section{MÉTODOS}

Este estudo epidemiológico transversal foi desenvolvido como parte integrante do projeto de pesquisa denominado "Estilos de Vida e Comportamentos de Risco à Saúde em Estudantes do Ensino Médio no Estado de Pernambuco”, cuja coleta de dados foi realizada no período de abril a outubro de 2006. O projeto de pesquisa que derivou a realização deste estudo foi aprovado, em julho de 2005, pelo Comitê de Ética em Pesquisa com Seres Humanos do Hospital Agamenon Magalhães, na cidade do Recife.

A população alvo do estudo foi limitada aos adolescentes (14 a 19 anos de idade), estudantes da rede pública estadual de ensino médio do estado de Pernambuco. Considerando-se todas as dependências administrativas (federal, estadual, municipal e privada), os dados fornecidos pela Secretaria de Educação do Esta- 
do indicavam que, em 2005, os estudantes matriculados na rede pública estadual representavam cerca de $80 \%$ do total de estudantes do ensino médio em Pernambuco. $\mathrm{O}$ dimensionamento amostral foi então efetuado de forma a atender os diversos objetivos do projeto, que incluíam a avaliação da exposição a dez fatores de risco à saúde, dentre os quais os indicadores de violência.

Para o dimensionamento amostral foram utilizados os seguintes critérios: população estimada em 353 mil estudantes; intervalo de confiança de 95\%; erro amostral de 3 pontos percentuais; prevalência estimada em 50\%; e, efeito de delineamento amostral estabelecido em quatro vezes o tamanho mínimo da amostra. Isto representaria uma amostra mínima com 4.217 estudantes.

Procurou-se garantir que a amostra selecionada representasse a população alvo quanto à distribuição conforme região geográfica, porte das escolas e período de matrícula dos estudantes (diurno e noturno). A distribuição regional foi observada pelo número de escolas existentes em cada uma das 17 GEREs (Gerências Regionais de Ensino). A fim de subsidiar o planejamento amostral, as escolas foram classificadas em três categorias: pequeno porte (menos de 200 estudantes); médio porte (200 a 499 estudantes); e, grande porte (500 estudantes ou mais). Os estudantes matriculados no período da manhã e da tarde foram agrupados numa única categoria (período diurno).

Para seleção da amostra requerida, recorreu-se a um procedimento de amostragem aleatória estratificada em dois estágios, sendo que a "escola" e a "turma" representaram as unidades amostrais, respectivamente, no primeiro e no segundo estágio. Todas as escolas da rede pública estadual em Pernambuco foram consideradas elegíveis para inclusão no estudo.

O questionário utilizado para coleta de dados (Global School-based Student Health Survey) foi autoadministrado em sala de aula sem a presença dos professores, sendo que todos os estudantes presentes foram convidados a participar, independentemente da idade. Além disso, o questionário não continha qualquer tipo de identificação pessoal (nome, matrícula). Os estudantes foram continuamente assistidos pelos aplicadores (sempre dois por turma) para que pudessem esclarecer dúvidas e auxiliar no preenchimento das informações. A participação dos estudantes foi voluntária e anônima, adotando-se a utilização de termo negativo de consentimento (parental passive consent form) para aqueles menores de idade.

Um estudo piloto foi conduzido antes do inicio da coleta de dados, a fim de determinar indicadores de reprodutibilidade de medidas e testar a aplicabilidade do instrumento. Os dados para o estudo piloto foram coletados em duas escolas da rede pública municipal de ensino na Cidade do Recife, com uma amostra de 138 adolescentes com idade de 14 a 19 anos (59 moças). Indicadores de reprodutibilidade (consistência de medidas teste-reteste) foram de moderados a altos na maioria dos itens do instrumento. Maiores detalhes sobre os procedimentos metodológicos adotados no desenvolvimento deste estudo estão disponíveis em publicações prévias ${ }^{5,13}$.

Dois indicadores de violência (envolvimento em episódios de violência física como vitima e envolvimento em brigas) foram analisados neste estudo e definidos operacionalmente como variáveis dependentes. "Envolvimento em episódios de violência física como vitima” foi determinado mediante análise das respostas à questão "Durante os últimos 12 meses, quantas vezes você sofreu algum tipo de violência física?". Similarmente "envolvimento em brigas" foi identificado pela pergunta "Durante os últimos 12 meses, quantas vezes você esteve envolvido numa briga?”. A variável independente no estudo foi a participação nas aulas de 
Educação Física, definida pelo número de aulas semanais que os estudantes relataram participar considerando três possibilidades de resposta $(0,1$ e 2 ou mais aulas/semana).

A idade dos estudantes foi dicotomizada nas seguintes faixas etárias: 14-16 e 17-19 anos. A cor da pele/etnia foi autorreferida considerando seis possíveis respostas (branca, preta, parda, indígena, amarela e outra) e, em seguida, agrupada em duas categorias (branco e não branco). A variável "morar com os pais" foi dicotômica ( $\operatorname{sim} /$ não) e somente os estudantes que referiram morar com pai e mãe foram classificados na categoria "sim”. Exposição a consumo de álcool também foi avaliada e todos os estudantes que relataram ter consumido qualquer tipo de bebida alcoólica em pelo menos um dos últimos 30 dias foram considerados expostos. Estes fatores serviram à caracterização da amostra e ajustamento das análises de associação.

As análises estatísticas foram realizadas mediante utilização do programa SPSS para Windows (versão 10). Enquanto que as análises descritivas foram efetuadas a partir da distribuição de frequências, nas análises bivariáveis recorreu-se à aplicação do teste de Qui-quadrado. Análises de associação entre as variáveis independentes e dependentes foram conduzidas mediante utilização de regressão logística separadamente para cada uma das variáveis dependentes (indicadores de violência). Inicialmente, procederam-se análises brutas e, em seguida, as análises multivariáveis que foram conduzidas a fim de controlar potenciais fatores de confusão, sendo que tanto as análises brutas quanto as ajustadas foram estratificadas por sexo devido à identificação de interação.

\section{RESULTADOS}

Para desenvolvimento do presente estudo foram utilizados dados de estudantes adolescentes de 76 escolas ( $11 \%$ do total de escolas estaduais do estado), distribuídas em 44 municípios, o que representa 23\% do total de municípios Pernambucanos. Foram entrevistados 4.210 estudantes com idade entre 14 e 19 anos (59,8\% do sexo feminino), sendo que dos estudantes convidados a participar do estudo 83 se recusaram ( $1,9 \%$ de recusas). O detalhamento das características demográficas e socioeconômicas dos participantes está apresentado na Tabela 1.

Para atingir o tamanho amostral definido a priori $(\mathrm{n}=4.217)$ mediante utilização de amostragem por conglomerados foram sorteadas 234 turmas de ensino médio (os conglomerados). Estimou-se que isto representaria uma amostra final, no mínimo, $10 \%$ superior ao inicialmente previsto, o que seria desejável devido às possíveis perdas e recusas. $\mathrm{Na}$ fase de coleta de dados, verificou-se que o número de estudantes com idade correspondente à faixa etária alvo foi inferior ao estimado, próximo a 18 por turma em média. Além das recusas, esta foi a principal razão para o menor número de estudantes na amostra final em relação ao previsto.

A prevalência de "envolvimento em brigas" e de "envolvimento em episódios de violência física como vitima” foi, respectivamente, de 21,2\% (IC95\%: 20,0; 22,5) e 10,7\% (IC95\%: 9,8; 11,7), significativamente superior entre os rapazes em comparação às moças (Figura 1). Verificou-se que 64,9\% (IC95\%: 63,4; 66,3) dos estudantes relataram não participar das aulas de educação física, sendo que esta proporção foi significativamente maior entre as moças (Figura 2). 
Tabela 1 - Características demográficas e socioeconômicas da amostra, estratificada por sexo $(n=4.210)$.

\begin{tabular}{|c|c|c|c|c|c|c|}
\hline \multirow{2}{*}{ Variável } & \multicolumn{2}{|c|}{ Rapazes } & \multicolumn{2}{|c|}{ Moças } & \multicolumn{2}{|c|}{ Todos } \\
\hline & $\%$ & $\mathrm{n}$ & $\%$ & $\mathrm{n}$ & $\%$ & $\mathrm{n}$ \\
\hline \multicolumn{7}{|l|}{ Idade (anos) * } \\
\hline $14-16$ & 35,5 & 599 & 46,4 & 1.167 & 42,0 & 1.766 \\
\hline $17-19$ & 64,5 & 1.089 & 53,6 & 1.346 & 58,0 & 2.435 \\
\hline \multicolumn{7}{|l|}{ Cor da pele/etnia $†$} \\
\hline Branco (a) & 24,8 & 417 & 25,5 & 639 & 25,2 & 1.056 \\
\hline Não branco(a) & 75,2 & 1.263 & 74,5 & 1.868 & 74,8 & 3.131 \\
\hline \multicolumn{7}{|l|}{ Mora com os pais ₹ } \\
\hline Sim & 68,1 & 1.131 & 60,6 & 1.512 & 63,6 & 2.643 \\
\hline Não & 31,9 & 529 & 39,4 & 981 & 36,4 & 1.510 \\
\hline \multicolumn{7}{|l|}{ Local de residência § } \\
\hline Urbana & 78,1 & 1.312 & 79,6 & 1.985 & 79,0 & 3.297 \\
\hline Rural & 21,9 & 367 & 20,4 & 510 & 21,0 & 877 \\
\hline \multicolumn{7}{|l|}{ Escolaridade materna II } \\
\hline$\leq 8$ anos de estudo & 69,4 & 1.087 & 74,5 & 1.772 & 72,5 & 2.859 \\
\hline 9-11 anos de estudo & 22,5 & 352 & 20,2 & 481 & 21,1 & 833 \\
\hline$\geq 12$ anos de estudo & 8,1 & 127 & 5,3 & 126 & 6,4 & 253 \\
\hline \multicolumn{7}{|l|}{ Situação ocupacional ๆ } \\
\hline Não trabalha & 69,3 & 1.158 & 84,8 & 2.121 & 78,6 & 3.279 \\
\hline Trabalha & 30,7 & 514 & 15,2 & 381 & 21,4 & 895 \\
\hline \multicolumn{7}{|l|}{ Turno ** } \\
\hline Diurno & 53,9 & 909 & 60,1 & 1.508 & 57,6 & 2.417 \\
\hline Noturno & 46,1 & 778 & 39,9 & 1.002 & 42,4 & 1.780 \\
\hline \multicolumn{7}{|l|}{ Série †† } \\
\hline $1^{\circ}$ ano & 46,2 & 779 & 43,8 & 1.101 & 44,8 & 1.880 \\
\hline $2^{\circ}$ ano & 30,9 & 522 & 32,6 & 818 & 31,9 & 1.340 \\
\hline $3^{\circ}$ ano & 22,9 & 386 & 23,6 & 593 & 23,3 & 979 \\
\hline
\end{tabular}

Dados missing: ${ }^{*} n=9,+n=23, \neq n=57, \S n=36, \| n=265,9 n=36,{ }^{*} n=13$, t十 $n=11$.

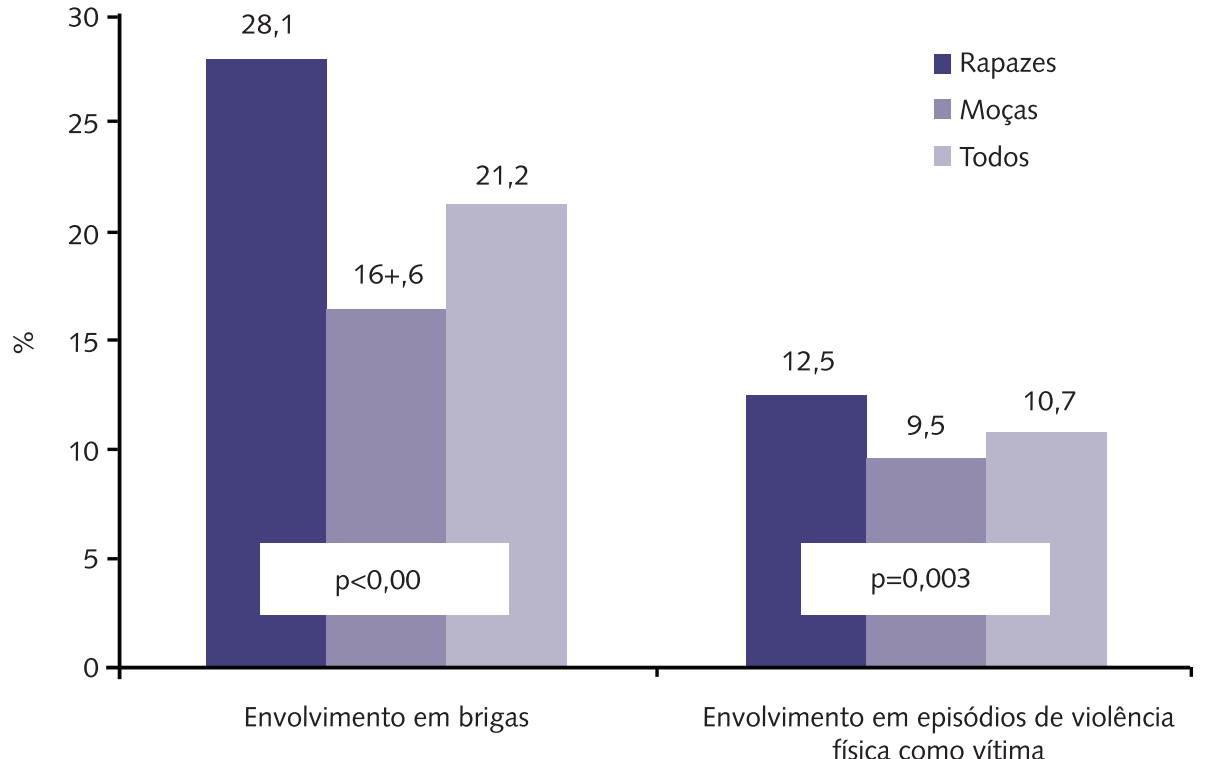

Figura 1 - Prevalência de indicadores de violência em adolescentes estudantes do ensino médio por sexo. Estado de Pernambuco, Brasil, 2006. 


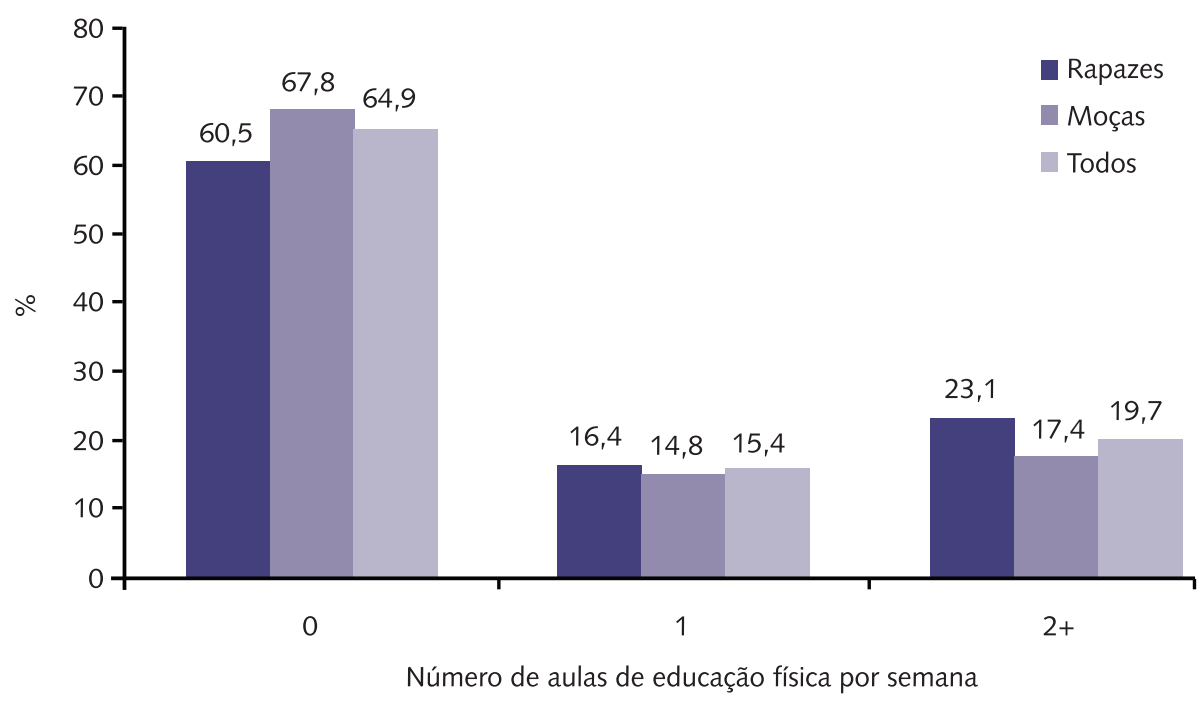

Figura 2 - Distribuição absoluta e relativa de adolescentes estudantes do ensino médio segundo participação nas aulas de Educação Física e sexo. Estado de Pernambuco, Brasil, 2006.

Quanto à análise de associação entre a participação nas aulas de educação física e os dois indicadores de violência considerados neste estudo, verificou-se que tanto em rapazes quanto nas moças a participação nas aulas de educação física foi um fator diretamente associado a envolvimento em brigas. Este resultado permaneceu praticamente inalterado mesmo após ajustamento para potenciais fatores de confusão (idade, turno, cor da pele, uso de bebidas alcoólicas e viver com os pais). A chance de relatar envolvimento em brigas foi $55 \%$ maior entre rapazes que participavam de duas ou mais aulas de educação física por semana em comparação àqueles que não participavam ( 0 aulas/semana). Entre as moças, esta chance foi $42 \%$ superior (ver resultados na Tabela 2 ).

Tabela 2 - Razão de odds (OR) bruto e ajustado e intervalos de confiança de 95\% para indicadores de violência, comparando estudantes que participam das aulas de Educação Física em relação àqueles que não participam (categoria de referência).

\begin{tabular}{|c|c|c|c|c|c|c|}
\hline Sexo & Indicador de violência & $\mathrm{N}^{\circ}$ aulas de $\mathrm{EF}$ & OR bruto (IC95\%) & Valor $\mathrm{p}$ & OR ajustado (IC95\%)* & Valor $p$ \\
\hline \multirow[t]{6}{*}{ Rapazes } & Envolvimento em episódios de & 0 & 1 & 0,60 & 1 & 0,62 \\
\hline & violência física como vitima & 1 & $1,10(0,74-1,65)$ & 0,64 & $1,14(0,74-1,76)$ & 0,55 \\
\hline & & 2 & $1,19(0,84-1,68)$ & 0,32 & $1,19(0,82-1,75)$ & 0,36 \\
\hline & Envolvimento em brigas & 0 & 1 & 0,00 & 1 & 0,02 \\
\hline & & 1 & $1,28(0,95-1,71)$ & 0,11 & $1,39(1,01-1,91)$ & 0,04 \\
\hline & & 2 & $1,55(1,20-2,00)$ & 0,00 & $1,45(1,09-1,93)$ & 0,01 \\
\hline \multirow[t]{6}{*}{ Moças } & Envolvimento em episódios de & 0 & 1 & 0,22 & 1 & 0,17 \\
\hline & violência física como vitima & 1 & $0,96(0,64-1,42)$ & 0,82 & $1,01(0,66-1,55)$ & 0,97 \\
\hline & & 2 & $1,32(0,95-1,85)$ & 0,10 & $1,40(0,98-2,01)$ & 0,07 \\
\hline & & 0 & 1 & 0,00 & 1 & 0,00 \\
\hline & Envolvimento em brigas & 1 & $0,77(0,55-1,07)$ & 0,12 & $0,75(0,52-1,09)$ & 0,13 \\
\hline & & 2 & $1,42(1,09-1,85)$ & 0,01 & $1,52(1,14-2,02)$ & 0,01 \\
\hline
\end{tabular}

* Ajustamento para idade, turno das aulas, cor da pele (branca/não branca), viver com os pais e exposição a consumo de bebidas alcoólicas.

\section{DISCUSSÃO}

O objetivo do presente estudo foi analisar a associação entre a participação nas aulas de educação física e indicadores de violência em adolescentes estudantes do ensino médio. Nas análises foram considerados dois indicadores de violência: o "envolvimento em brigas" e o "envolvimento em episódios de violência física 
como vitima". O principal achado neste estudo foi a identificação de que, em 2006 e entre os adolescentes estudantes do ensino médio no estado de Pernambuco, a participação nas aulas de educação física foi um fator diretamente associado ao envolvimento em brigas, independentemente do gênero e de outros potenciais fatores de confusão, como a exposição ao uso de bebidas alcoólicas. Apesar de não ter sido objetivo do estudo, os resultados também revelaram que o envolvimento em brigas foi um indicador de violência mais prevalente que o envolvimento em episódios de violência como vítima.

Esta associação positiva entre a participação nas aulas de educação física e o envolvimento em brigas pode ser resultante da confusão provocada por outros fatores não controlados nas análises, a exemplo da participação dos sujeitos em esportes ou outras atividades físicas fora do contexto das aulas de educação física. As análises foram repetidas, acrescentando-se aos demais fatores de confusão o nível global de atividade física dos sujeitos. Os resultados permaneceram praticamente inalterados, com pequena variação apenas nos limites dos intervalos de confiança para odds ratio.

Este estudo tem limitações que precisam ser destacadas. Primeiro, os dados foram autorrelatados e o viés de resposta pode ter conduzido a subestimação da prevalência de indicadores de violência e exposição a consumo de bebidas alcoólicas. Procurou-se contornar esta última limitação mediante utilização de um questionário não identificado (anônimo) e pela garantia do sigilo das informações fornecidas. Além disso, os professores da escola não permaneciam na sala de aula durante a aplicação do questionário aos estudantes.

A amostra não abrangia adolescentes que estão matriculados na rede privada de ensino e aqueles que estão fora do sistema escolar, por isso, mesmo considerando que o estudo foi desenvolvido com uma amostra relativamente grande, a generalização dos resultados deve ser limitada à população alvo do estudo e ao local de realização do estudo (estado de Pernambuco). Justifica-se, contudo, que a decisão de não incluir escolas do sistema privado de ensino no planejamento amostral foi decorrente da constatação de que mais de $80 \%$ dos adolescentes matriculados neste nível de ensino estão em escolas públicas estaduais. Deve-se destacar ainda que os resultados relatados neste estudo foram extraídos da análise secundária de levantamento realizado em 2006, portanto, admite-se que tanto a prevalência de participação nas aulas de Educação Física quanto dos indicadores de violência pode ter modificado ao longo deste período.

Apesar do delineamento transversal do estudo, a possibilidade de viés de causalidade reversa é pequena, pois é pouco plausível admitir que os alunos tenham maior chance de participação nas aulas de educação física por terem igualmente maior envolvimento em episódios de violência. Mesmo assim, sugere-se cautela ao interpretar as associações que foram identificadas como sendo indicativas de efeito. Além disso, outros fatores podem explicar o envolvimento em brigas e a impossibilidade de controlar os mesmos no delineamento do estudo e nas análises pode ter também conduzido a viés de confusão.

Apesar das limitações mencionadas, o presente estudo tem pontos fortes que merecem ser destacados, em especial o fato de ter sido realizado com amostra representativa de uma população definida e com suficiente poder estatístico para as análises que foram propostas. Outro ponto positivo foi a inclusão no estudo de adolescentes residentes em área rural, que estão expostos de maneira diferente à violência interpessoal e constituem um subgrupo usualmente não incluído nos estudos devido à dificuldade de recrutamento. Além disso, a coleta dos dados foi realizada com um instrumento que foi previamente testado e apresentou bons indicadores de 
reprodutibilidade teste-reteste para medida das variáveis observadas neste estudo.

A participação em esportes, particularmente naqueles considerados agressivos, é apontada como um dos inúmeros fatores predisponentes que se situam numa cadeia causal complexa e que explica a violência em adolescentes. Tais fatores guardam entre si interações e efeitos de moderação que precisam ser considerados quando da realização das análises. Por exemplo, a exposição a consumo de bebidas alcoólicas na adolescência e o gênero são variáveis que podem confundir e atuar como moderadores na análise da associação entre a participação nas aulas de educação física. Assim, a possibilidade de ajustamento das análises (consumo de bebidas alcoólicas) e de estratificação (gênero) constituem pontos positivo do presente estudo.

$\mathrm{Na}$ revisão de literatura conduzida para desenvolvimento deste estudo, nenhuma investigação foi localizada focalizando a associação entre a participação nas aulas de educação física e indicadores de violência em adolescentes, o que dificulta a comparação dos resultados. Mas, alguns estudos foram conduzidos a fim de explorar a associação entre a participação em esportes ${ }^{14} \mathrm{e}$ em outras atividades físicas ${ }^{15}$, dentro e fora do ambiente escolar, com a ocorrência de indicadores de violência.

Estudos anteriores evidenciaram que as frequências de participação em esportes ${ }^{16}$ e de envolvimento em episódios de violência ${ }^{17}$ são maiores entre os rapazes, sendo que a diferença intersexual quanto ao envolvimento em violências foi observada também neste estudo. Assim, esperava-se observar uma discrepância intersexual no tocante à associação entre a participação nas aulas de educação física e os indicadores de violência, hipótese que não se confirmou diante dos achados relados no presente estudo.

Assim como em outros países, nas escolas brasileiras a violência vem se tornando um problema de grande magnitude ${ }^{18}$. Paralelamente, cresce o corpo de evidências de que grande proporção de adolescentes está exposta a condutas de risco à saúde ${ }^{19}$. Diante deste cenário, organizações científicas e sanitárias ${ }^{20}$, assim como pesquisadores ${ }^{12}$, têm indicado que as aulas de Educação Física podem ter um papel fundamental no desenvolvimento de intervenções em saúde ou na concretização de políticas públicas como o Programa Saúde na Escola do Ministério da Saúde ${ }^{21}$.

A participação nas aulas de Educação Física está associada a maior nível de atividade física global ${ }^{22,5}$ e hábitos alimentares mais saudáveis ${ }^{5}$. Além disso, intervenções para promoção de atividade física que são baseadas em modificação do currículo das aulas de Educação Física tem se mostrado efetivas em aumentar o nível de atividade física de adolescentes ${ }^{23}$. Todavia, no contexto de vida real, considerando as diferentes características que as aulas de educação física podem assumir, é possível levantar a hipótese de que estas podem estar associadas também a desfechos negativos em saúde como o que foi observado no presente estudo.

A associação direta entre a participação nas aulas de educação física e o envolvimento em brigas, observada no presente estudo tanto entre rapazes quanto entre as moças, ao menos em parte, pode ser explicada pela acentuada ênfase na prática assistemática de esportes que ocorre nas aulas de educação física. A plausibilidade desta ligação reside nas evidências disponíveis sugerindo que a participação em esportes e, em particular, de algumas modalidades esportivas, constituem um fator de risco para envolvimento em episódios de violência interpessoal ${ }^{8,9,14}$.

Os resultados deste estudo têm importantes implicações para gestores das áreas de esporte, educação e saúde, na medida em que apontam a necessidade de revisão da proposta curricular e da prática pedagógica na Educação Física escolarizada. Isso decorre da plausível associação entre a participação nas aulas de Educação Física e o envolvimento em brigas que foi observada neste grupo de adolescen- 
tes estudantes do ensino médio. Também é particularmente importante quando o estudo realizado por Marshall e Hardman (2000) ${ }^{24}$ aponta que em países da América latina, a exemplo do que ocorre em todo o mundo, cerca de $67 \%$ dos pais e 88\% dos professores de outras disciplinas curriculares não apóiam ou são indiferentes em relação às aulas de Educação Física. Há, sem dúvida, uma pressão cada vez maior para redução do tempo destinado às aulas de Educação Física, algo que se contrapõem às diretrizes tanto de agências internacionais ${ }^{25}$ quanto nacionais ${ }^{21}$.

Para pesquisadores da área da Educação Física fica a clara mensagem de que é preciso investigar mais e contribuir para melhorar a qualidade das aulas de Educação Física. Por outro lado, considerando-se as características do presente estudo não se pode assumir que o oferecimento de aulas de Educação Física pode provocar aumento da frequência de violência nas escolas. Assume-se, portanto, que existe uma associação que precisa ser mais bem investigada e que pode estar circunscrita ao contexto em que o estudo foi desenvolvido, escolas públicas do estado de Pernambuco.

A natureza observacional e a abordagem quantitativa desta investigação revela a existência de uma inter-relação, mas não permite que sejam identificados os mecanismos e as razões pelas quais a participação nas aulas de educação física está associada à elevação da chance de que os estudantes adolescentes venham a se envolver em brigas. Esta lacuna precisará ser enfrentada em futuros estudos, possivelmente mediante uso de abordagens qualitativas de pesquisa. Dentre os desfechos focalizados no presente estudo não foram incluídos indicadores de outros tipos de violência, alguns dos quais com elevada frequência de ocorrência nesta faixa etária, como a violência verbal, emocional ou sexual. Como, até onde se tem conhecimento, este foi o primeiro estudo focalizando a associação entre a participação nas aulas de educação física e indicadores de violência na adolescência, os achados aqui relatados precisarão ser confirmados e o corpo de conhecimento ampliado mediante replicação, critica ou aperfeiçoamento do protocolo de investigação adotado no presente estudo.

Os resultados do presente estudo permitiram concluir que a participação nas aulas de Educação Física é um fator associado a um indicador específico de violência: o envolvimento em brigas. Este achado foi observado tanto entre os rapazes quanto entre as moças e independentemente da exposição a consumo de bebidas alcoólicas. Uma das hipóteses levantadas é de que esta ligação seja decorrente da acentuada ênfase na prática assistemática de esportes que ocorre nas aulas de educação física. Todavia, as razões ou os mecanismos que explicam a associação verificada no presente estudo ainda não estão suficientemente esclarecidos, devendo ser alvo de futuras investigações.

\section{Financiamento}

Estudo apoiado com auxílio financeiro da Coordenação de Aperfeiçoamento de Pessoal de Nível Superior, do Conselho Nacional de Desenvolvimento Científico e Tecnológico e da Fundação de Amparo à Ciência e Tecnologia do Estado de Pernambuco.

\section{REFERÊNCIAS}

1. Taliaferro LA, Rienzo BA, Donovan KA. Relationships between youth sport participation and selected health risk behaviors from 1999 to 2007. J Sch Health. 2010;80:399-410.

2. Moore MJ, Werch CE. Sport and physical activity participation and substance use among adolescents. J Adolesc Health.2005;36:486-93. 
3. Myers L, Strikmiller PK, Webber LS, Berenson GS. Physical and sedentary activity in school children grades 5-8: the Bogalusa Heart Study. Med Sci Sports Exerc. 1996;28:852-59.

4. Pate RR, Ward DS, O'Neill JR, Dowda M. Enrollment in physical education is associated with overall physical activity in adolescent girls Res QExerc Sport.2007;78:265-70.

5. Tassitano RM, Barros MV, Tenório MC, Bezerra J, Florindo AA, Reis RS. Enrollment in physical education is associated with health-related behavior among high school students. J Sch Health. 2010;80:126-33.

6. Minamisava R, Nouer SS, Morais Neto OL, Melo LK, Andrade ALSS. Spatial clusters of violent deaths in a newly urbanized region of Brazil: highlighting the social disparities Int J Health Geogr. 2009; 8:66.

7. Assis SG, Avanci JQ, Pesce RP, Ximenes LF. Situação de crianças e adolescentes brasileiros em relação à saúde mental e à violência. Ciênc. Saúde Coletiva: 2009;14:349-361.

8. Garry JP, Morrissey SL. Team sports participation and risk taking behaviors among a biracial middle school population. Clin J Sport Med. 2000;10:185-90.

9. Pate RR, Trost SG, Levin S, Dowda M. Sports participation and health-related behaviors among US youth. Arch Pediatr Adolesc Med. 2000;154:904-11.

10. Miller KE, Hoffman JH, Barnes GM, Farrell MP, Sabo D, Melnick MJ. Jocks, gender, race, and adolescent problem drinking. J Drug Educ. 2003; 33:445-62.

11. Guedes DP, Guedes JERP. Characteristic of the school physical education programs. Rev Paul Educ Fi. 1997; 11:49-62.

12. Hoehner CM, Soares J, Parra Perez D, Ribeiro IC, Joshu CE, Pratt M, et al. Physical activity interventions in Latin America: a systematic review. Am J Prev Med. 2008; 34:224-233.

13. Santos CM,Wanderley Júnior RS, Honda Barros SS, Farias Júnior JC, Barros MVG. Prevalência e fatores associados à inatividade física nos deslocamentos para escola em adolescentes. Cad. Saúde Pública. 2010;26:1419-430.

14. Forbes GB, Adams-Curtis LE, Pakalka AH, White KB. Dating aggression, sexual coercion, and aggression-supporting attitudes among college men as a function of participation in aggressive high school sports. Violence Against Women. 2006;12:441-55.

15. Turagabeci AR, Nakamura K, Takano T. Healthy lifestyle behaviour decreasing risks of being bullied, violence and injury. PLoS One. 2008; 3:e1585.

16. Deaner RO, Geary DC, Puts DA, Ham SA, Kruger J, Fles E, et al. A Sex Difference in the Predisposition for Physical Competition: Males Play Sports Much More than Females Even in the Contemporary U.S. PLoS ONE. 2012; 7: e49168.

17. Hanson RF, Borntrager C, Self-Brown S, Kilpatrick DG, Saunders BE, Resnick HS, et al. Relations among Gender, Violence Exposure, and Mental Health: The National Survey of Adolescents. Am J Orthopsychiatry. 2008; 78:313-21.

18. Waiselfisz JJ. Mapa da violência 2011: os jovens do Brasil. São Paulo: Instituto Sangari; Brasília, DF: Ministério da Justiça, 2011.

19. Farias Júnior JC, Nahas MV, Barros MVG, Loch MR, Oliveira ESA, De Bem MFL, et al. Comportamentos de risco à saúde em adolescentes no Sul do Brasil: prevalência e fatores associados. Rev Panam Salud Publica. 2009;25:344-52.

20. Center for Disease Control and Prevention. Participation in high-school physical educationUnited States 1991-2003. Morbidity and Mortality Weekly Report., 2004.

21. Brasil. Avaliação de Efetividade de Programas de Atividade Física no Brasil. Brasília: Ministério da Saúde. Secretaria de vigilância em saúde, 2011.

22. Trudeau F, Shephard RJ. Contribution of School Programmes to Physical Activity Levels and Attitudes in Children and Adults. Sports Med. 2005; 35:89-105.

23. Barros MVG, Nahas MV, Hallal PC, Farias Júnior JC, Florindo AA, Honda de Barros SS. Effectiveness of a school-based intervention on physical activity for high school students in Brazil: the Saude na Boa Project. J Phys Act Health. 2009 ; 6:163-9.

24. Marshall J, Hardman K. The state and status of physical education in schools in international context. European Physical Education Review. 2000; 6:203-219.

25. United States. Department of Health and Human Services. Healthy People 2010. Washington, DC, 2000.
Endereço para Correspondência Simone Storino Honda Barros Rua Arnóbio Marques, 310, Campus Universitário HUOC/ESEF Santo Amaro, Recife - PE, 50100-130 E-mail: sihonda.barros@gmail.com Telefone: +55 81 3183-3376

Recebido 16/10/2013 Revisado 31/10/2013 Aprovado 04/11/2013 